\title{
The Reliability of Neurological
} Measurement in the Vastus Medialis: Implications for Research and Practice

\section{OPEN ACCESS}

Edited by:

Costantino Balestra,

Haute École Bruxelles-Brabant

(HE2B), Belgium

Reviewed by:

Heidi Haavik,

New Zealand College of Chiropractic,

New Zealand

Giorgio Sandrini,

University of Pavia, Italy

${ }^{*}$ Correspondence:

Ashlee M. Hendy

a.hendy@deakin.edu.au

Specialty section:

This article was submitted to

Movement Science and Sport

Psychology,

a section of the journal

Frontiers in Psychology

Received: 16 July 2018 Accepted: 11 September 2018

Published: 01 October 2018

Citation:

Leung H, Latella C, Lamon S and

Hendy AM (2018) The Reliability

of Neurological Measurement in the Vastus Medialis: Implications

for Research and Practice.

Front. Psychol. 9:1857.

doi: 10.3389/fpsyg.2018.01857
Hans Leung, Christopher Latella, Séverine Lamon and Ashlee M. Hendy*

School of Exercise and Nutrition Sciences, Institute for Physical Activity and Nutrition (IPAN), Deakin University, Geelong, VIC, Australia

The integrity of the corticomotor pathway is paramount in the optimal functioning of skeletal muscle. However, variability of neurophysiological assessment via peripheral nerve and transcranial magnetic stimulation can render interpretation difficult. Seldom evidence exists regarding the reliability of such measurements in the leg extensors, which have important locomotive and functional roles. This study aimed to assess the test-retest reliability of peripheral, corticospinal and intracortical responses in the vastus medialis. Transcranial magnetic and direct current electrical nerve stimulation were delivered to sixteen healthy young adults (8M and 8F) on two separate occasions. The Hoffmann reflex, maximal compound wave, motor evoked potential, corticospinal silent period, intracortical facilitation, and short-interval intracortical inhibition were recorded from the vastus medialis at rest, and during controlled submaximal voluntary contraction. Relative reliability was quantified using intra-class correlation coefficient (ICC2,1). Absolute reliability was quantified using standard error of measurement (SEm) and minimal detectable change (MDC). Corticospinal silent period, corticospinal silent period/motor evoked potential ratio, active motor evoked potential, maximal Hoffman reflex, and passive short-interval intracortical inhibition demonstrated "good to excellent" relative reliability (ICC $\geq 0.643$ ). Intracortical facilitation demonstrated the lowest relative reliability (ICC $=0.420-0.908)$. Corticospinal silent period displayed the lowest absolute reliability (SEm $\leq 18.68 \%$ ). Good reliability of the maximal compound wave, Hoffman reflex, motor evoked potential, and corticospinal silent period allow for reliable neurological evaluation of peripheral and corticospinal pathways in the vastus medialis. Future research should investigate reliability of the intracortical (shortinterval intracortical inhibition and intracortical facilitation) measures by using different paired-pulse stimulus parameters. These findings hold important implications for neurophysiological assessment conducted in the leg extensor group.

Keywords: transcranial magnetic stimulation, corticospinal, H-reflex, leg extensors, inter-session

Abbreviations: CSP, corticospinal silent period; GABAA, gamma-aminobutyric acid A; HMAX, maximal Hoffman reflex; H-reflex, Hoffmann reflex; ICC, intra-class correlation coefficient; ICF, intracortical facilitation; MDC, minimal detectable change; MEP, motor evoked potential; MMAX, maximal compound wave; MVIC, maximal voluntary isometric contraction; NMDA, N-methyl-d-aspartate; SEm, standard error of measurement; SICI, short-interval intracortical inhibition; TMS, transcranial magnetic stimulation; VM, vastus medialis. 


\section{INTRODUCTION}

The integrity of the human motor system is essential in the development of muscular force, power and overall physical function. Stimulation of intracortical and corticospinal pathways is commonly used to provide insight into the behavior of excitatory and inhibitory networks underpinning motor control of movement. In particular, the musculature of the lower limbs play an important role in functional and performance tasks, locomotion and knee joint integrity (Avramidis et al., 2003; Peeler and Anderson, 2007). Therefore, the reliable assessment of the motor pathway associated with the lower limbs is paramount in clinical, research and human performance settings. In this research, we investigated the lower-limb muscle VM for two reasons; (1) the VM generates the most consistent H-reflex response compared to the VL and rectus femoris (Kameyama et al., 1989) and (2) the VM plays an important role in the function and injury prevention of the knee. The VM has a crucial influence on gait performance and motor control of the knee (Avramidis et al., 2003), and its weakness is viewed as a contributing factor to patellofemoral joint dysfunction (Peeler and Anderson, 2007). The delayed onset of EMG activity of the $\mathrm{VM}$ relative to the $\mathrm{VL}$, which indicate neurological dysfunction, is common in patients with patellofemoral pain syndrome (Cowan et al., 2001). Furthermore, the VM tends to atrophy to a greater extent than the other quadriceps muscles during disuse (Appell, 1990).

Peripheral nerve stimulation provides insight into the pre- or post-synaptic excitability of Ia afferent reflex pathways and/or the excitability of lower motor neurons (Pierrot-Deseilligny and Mazevet, 2000). This has been used to investigate musculoskeletal injury (Hopkins and Palmieri, 2004), neurological conditions (Braddom and Johnson, 1974), exercise based adaptations (Earles et al., 2002), and fatigue (Latella et al., 2017). The reliability of the H-reflex has generally been reported in the upper limbs (Miller et al., 1995; Bodofsky, 1999) and ankle flexors (Hopkins et al., 2000; Palmieri et al., 2002). There is, however, some debate around the reliability of these measures in the VM. Previously, "excellent" within-subject reliability has been reported in the VM across six subsequent days (Hopkins and Wagie, 2003), whilst others reported poor reliability due to high within-subject variation (Doguet and Jubeau, 2014). Calder et al. (2005) reported "excellent" reliability of the MMAX in the elbow flexors. Recently, Nuzzo et al. (2016) suggested a posture-dependent effect on MMAX, questioning the reliability of its use in various research protocols. In the lower limbs, Latella et al. (2017) reported "good" reliability of MMAX, recorded from the rectus femoris. However, this has not been comprehensively investigated in the VM.

Transcranial magnetic stimulation provides a non-invasive assessment of the entire motor pathway and is used extensively in clinical and research settings (Sacco et al., 1997). TMS can quantify temporal and spatial information (i.e., cortical, corticospinal, and spinal) with single- and paired-pulse stimulation techniques. Despite its widespread use, the evoked responses demonstrate moderate to large intra- and inter-tester and -participant variability (Orth and Rothwell, 2004; Cavaleri et al., 2017). In particular, factors such as circadian rhythm (Sale et al., 2007), age (Remaud et al., 2014; Mouthon et al., 2016), ovarian hormones (Inghilleri et al., 2004), fatigue (Taylor et al., 2006), and training status (Pearcey et al., 2014) can influence corticospinal excitability. Orth and Rothwell (2004) have previously reported that the inter-subject variation of the CSP of an intrinsic hand muscle is high, but is reduced when expressed as a ratio of the MEP. More commonly, the MEP/MMAX ratio is reported as an alternative means of normalization to reduce inter-subject variability. Further consideration must also be given to the influence of stimulation during passive rest or active contraction, with variability generally decreasing during muscular activation (Thomas et al., 2016). The reliability of the TMS has been well reported in the upper limbs (Maeda et al., 2002; Wassermann, 2002; Kamen, 2004; Ngomo et al., 2012), but is less common in the lower limbs. To our knowledge, only a handful of authors have established MEP reliability in the VM during isometric contraction (Luc et al., 2014; Temesi et al., 2017), whilst to our knowledge, passive MEP reliability has not been investigated. Additionally, no studies have concurrently investigated peripheral nerve and corticospinal measures in the VM. In paired-pulse protocols, Thomas et al. (2016) and Temesi et al. (2017) have reported "good" reliability of short and long interval cortical inhibition (SICI and LICI) in the VM. Although the authors also reported "good" reliability of ICF, this has not always been reported (Latella et al., 2017). Given that it is increasingly common for neurological studies to use single- and paired-pulse TMS as their outcome measures (Pearcey et al., 2014; Remaud et al., 2014; Hunter et al., 2016; Latella et al., 2016, 2017; Mouthon et al., 2016), the reliability of these measures should be established. Traditional TMS neurological study protocols have generally been developed for studies investigating intrinsic hand muscles, not for the lower limb. In particular, the disparate functional roles, fiber type and architectural composition of the muscles within the leg extensor group suggest that further research is warranted to elaborate on previous findings.

The aim of this study was to investigate the reliability of the neurophysiological responses in the VM. In particular, we aim to assess peripheral (H-reflex and $\mathrm{M}$-wave), corticospinal (MEP and CSP) and intracortical (SICI and ICF) measures evoked from peripheral nerve and TMS. The results of this study will help inform future neurophysiological studies investigating the VM and will hold important implications for future lower limb rehabilitation research and clinical practice.

\section{MATERIALS AND METHODS}

\section{Participants}

Sixteen (8 male, age: $25.88 \pm 3.83$ years, 8 female, age: $23.13 \pm 2.95$ years) healthy participants participated in the study. Written informed consent was obtained for each participant prior to the start of the study. Prior to TMS, all participants were screened using a TMS safety questionnaire to exclude potential participants with contraindications to neurological testing (Rossi, 2009). All procedures used in this study were approved by the Deakin University Human Research Ethics 
Committee (2017-023) and conducted to the standards set by the Declaration of Helsinki.

\section{Experimental Protocol}

Participants performed $2 \times 1 \mathrm{~h}$ testing sessions (Day 1 and Day 2), separated by a period of 7-10 days. All sessions were conducted at the same time of the day. Each session involved MVIC and, peripheral and transcranial nerve stimulation. The procedures for the pre and post testing were identical.

\section{Maximal Voluntary Isometric Contraction}

Maximal torque of the leg extensors was measured during a $3 \mathrm{~s}$ MVIC. Participants performed three MVIC trials (2 s ramp up and $3 \mathrm{~s}$ maximal contraction), separated by a $60 \mathrm{~s}$ rest period. Trials were conducted with the participants seated upright on an isokinetic dynamometer (Biodex system 4 pro, Biodex medical systems, United States). An immovable leg extension arm was secured approximately $2 \mathrm{~cm}$ above the medial malleolus (Krishnan et al., 2011) and the ankle of the right foot strapped in place. The hip was positioned at $90 \mathrm{o}$ of flexion with a $60 \mathrm{o}$ flexion angle of the right knee. Strong verbal encouragement was provided to the participant by the researchers. The maximal peak torque $(\mathrm{Nm})$ of the three trials was recorded as the MVIC.

\section{Surface Electromyography}

Surface electromyography activity was recorded from the VM muscle in the right leg using bipolar Ag-AgCl electrodes. Two electrodes positioned $20 \mathrm{~mm}$ apart were placed over the muscle belly of the VM at the $80 \%$ mark of the line between the anterior spina iliaca superior and the joint space in front of the anterior border of the medial ligament (Hermens et al., 2000; Fratini et al., 2009). The participants' skin was shaved and cleaned with an isopropyl alcohol swab prior to electrode placement to ensure a clear signal was obtained. Surface electromyography activity signals were analyzed offline using PowerLab 4/35 (ADinstruments, Australia).

\section{Peripheral Nerve Stimulation}

The H-reflex and M-wave were evoked via electrical stimulation of the right femoral nerve using a constant current stimulator (Digitimer, Hertfordshire, SDR Scientific). To maintain a consistent posture (Cecen et al., 2018), participants laid supine, with their leg resting passively and in a slightly flexed, supported position. Nerve stimulation (pulse width $1 \mathrm{~ms}$ ) was delivered with bipolar electrodes placed over the femoral triangle approximately 3-5 cm below the inguinal ligament along the right inguinal fold (Doguet and Jubeau, 2014). The stimulus intensity was increased until the peak-to-peak amplitude of the M-wave became saturated (MMAX). Figure 1 depicts an example of a MMAX obtained from one of our participants, represented by Figure 1A. The stimulus intensity was then increased by a further $20 \%$ to ensure a supramaximal stimulus was delivered each time. The same procedure was applied for active MMAX measures, but with participants flexing their right knee at a $60 \mathrm{o}$ angle (full knee extension equates to $0 \mathrm{o}$ ), and contracting at $10 \%$ of MVIC.

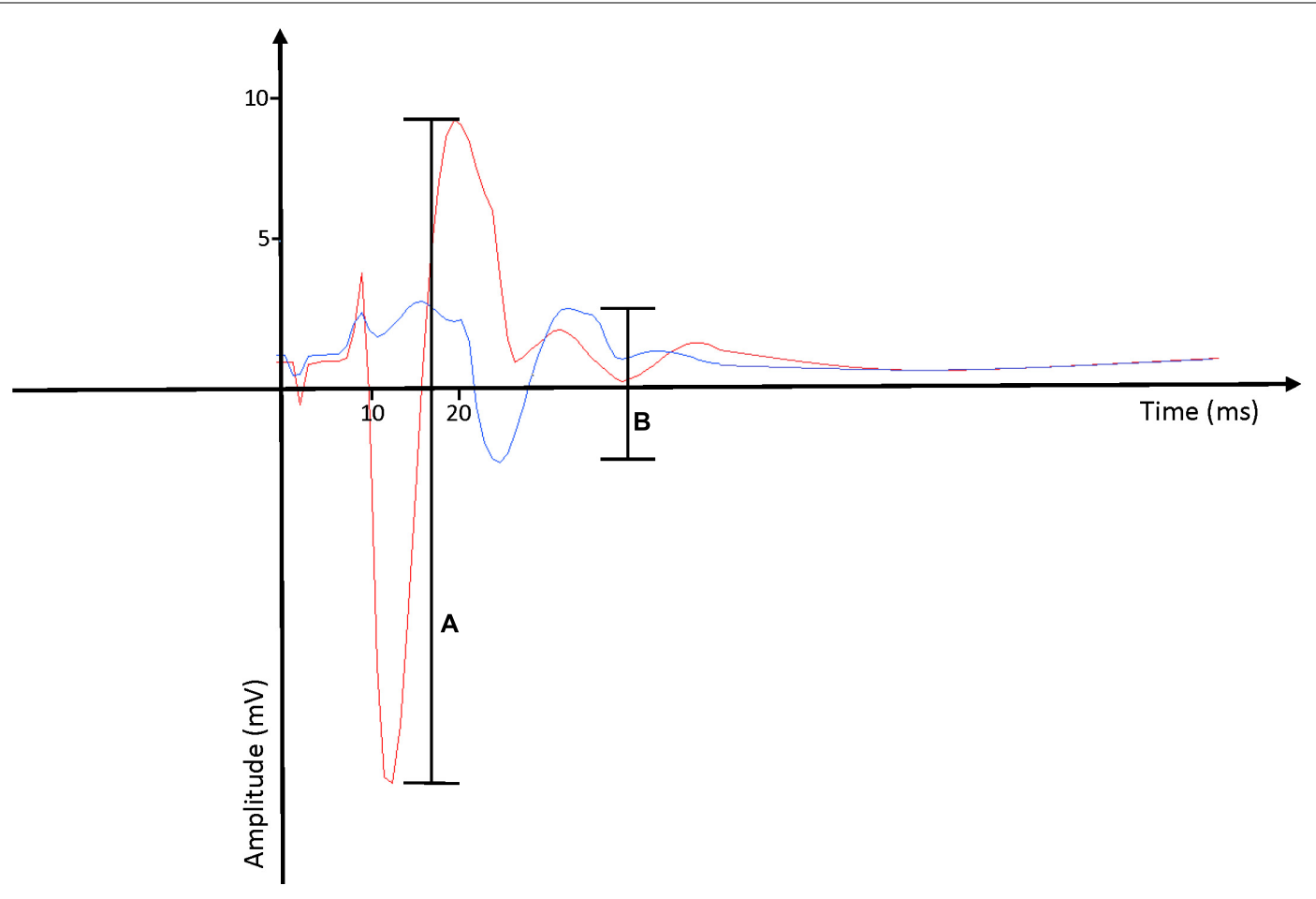

FIGURE 1 | Examples of (A) MMAX response and (B) H-reflex response, as measured by surface electromyography recordings. MMAX response typically occurs at around the 9 ms mark. H-reflex response typically occurs at around the 15-20 ms mark. 
To assess the H-reflex, the stimulus intensity was initially reduced to a point where no response was observed, then increased at increments of $2.5 \mathrm{~mA}$ until the largest peakto-peak response. Offline examination of the wave-form was conducted manually with a cursor, and the largest amplitude was reported as the $\mathrm{H}_{(\mathrm{MAX})}$. This value was also used to determine the $H_{(M A X)}: M_{(M A X)}$ ratio (Doguet and Jubeau, 2014). The H-reflex was observed approximately 15-25 ms after stimulation (Palmieri et al., 2004). Figure 1 depicts an example of a HMAX response from one of our participants, represented by Figure 1B. Successive stimuli were separated by a $20 \mathrm{~s}$ inter-stimulus interval to minimize any potentiation and/or fatigue (Hopkins and Wagie, 2003).

\section{Transcranial Magnetic Stimulation}

All TMS was delivered with the participant seated upright on the isokinetic dynamometer. For passive conditions, participants were instructed to relax their right leg in the seated position, whilst for active measures, participants were instructed to hold at a steady $10 \%$ MVIC using real time visual feedback. Both conditions were conducted at $60^{\circ}$ of flexion. TMS was delivered over the motor cortex (M1) using a $110 \mathrm{~mm}$ concave doublecone coil (Magstim Co., United Kingdom) attached to a BiStim 2002 magnetic stimulator (Magstim Co., United Kingdom). The coil was positioned horizontally in an anterior-posterior direction while delivering the stimulus. Participants wore a tight fitting cap (EasyCap, Germany), positioned with reference to the nasion-inion and interaural lines to ensure intra- and intertest consistency of the coil placement. To locate the optimal site, stimuli were delivered over various points along the M1. The optimal site was the location on the M1 that evoked the greatest MEP amplitude in the right VM. This spot was then marked down on the cap and used for further testing. The resting motor threshold (RMT) and active motor threshold (AMT) were defined as the lowest stimulus intensity required to elicit an MEP of at least 50 or $200 \mu \mathrm{V}$, respectively, in at least six out of 10 trials (Rothwell et al., 1999). Corticospinal excitability was determined by the peak-to-peak amplitude of the single pulse waveform at a stimulus intensity of 1.2 times AMT and RMT from 10 trials (Chen, 2000). The CSP was calculated as the duration in ms from the onset of the MEP to the return of pre-stimulation surface electromyography activity level.

Paired-pulse stimulation was used to assess intracortical inhibition and facilitation. Ten pairs of conditioning- and test-pulses were delivered at $80 \%$ and $120 \%$ of RMT, respectively.
The inter-stimulus interval was set at 3 and $12 \mathrm{~ms}$ for SICI and ICF, respectively (Wassermann, 2008; Rossini et al., 2015). Passive conditions were investigated for both SICI and ICF and an additional active condition was investigated for SICI. Both SICI and ICF were expressed as a percentage of the average single-pulse MEP (Wassermann, 2008; Rossini et al., 2015).

\section{Statistical Analysis}

All data was analyzed using IBM SPSS Statistics v.25 (IBM, United States). Test-retest reliability was then analyzed for each outcome measure (MVIC, MEP, MEP/MMAX, CSP, CSP/MEP, ICF and SICI, and MMAX and HMAX). Relative overall reliability was assessed by calculating the two-way mixed effects, absolute agreement, single measurement ICC2,1 (Koo and Li, 2016). ICCs were classified as "poor" $(<0.40)$, "fair" $(0.40-$ $0.59)$, "good" (0.60-0.74), and "excellent" ( $\geq 0.75)$ (Temesi et al., 2017). The within participant coefficient of variation (CV) was expressed as the average of each individual's $\mathrm{CV}$, a percentage derived from the formula $(\mathrm{SD} / \mathrm{Mean}) \times 100$. CV values $\leq 10 \%$ indicate low variability. Absolute reliability was calculated to establish the variability of repeated measurements (Atkinson and Nevill, 1998) using the Standard error of measurement $(S E m)=$ $S D \sqrt{1-I C C}$ (Weir, 2005). The MDC was calculated via the formulae $M D C=S E M \times 1.96 \times \sqrt{2}$, to determine to minimum difference required between trials for the change to be considered real (Weir, 2005). Bland-Altman plots were used to visualize the agreement between the two trials. 95\% limits of agreement (LOA) was calculated via the formulae $x \pm 1.96 \times S D$, where $\mathrm{x}$ is the mean difference between the two trials, and SD is the standard deviation of differences of the trials. The smaller the range of the LOAs, the better the agreement is.

\section{RESULTS}

\section{Maximal Voluntary Isometric Contraction}

Table 1 displays the reliability of the MVIC measurements. The relative reliability (ICC $=0.945,95 \%$ CI $[0.852-0.980]$ ) was “excellent." The SEm, CV, and MDC were 19.8, 8.30\%, and 55.0, respectively.

\section{Peripheral Nerve Stimulation}

Reliability data of peripheral nerve measures are presented in Table 1. Figure 2 depicts the comparison of individual measurements across the two trials. Passive MMAX had "fair to

TABLE 1 | Reliability data for all peripheral measures across two testing sessions.

\begin{tabular}{|c|c|c|c|c|c|c|c|}
\hline & Day I & Day 2 & ICC & $95 \% \mathbf{C l}$ & SEm (\%) & CV\% & MDC \\
\hline $\operatorname{MVIC}(\mathrm{N})$ & $163.13 \pm 58.66$ & $165.88 \pm 60.84$ & 0.945 & $0.852-0.980$ & $19.8(12.05)$ & 8.30 & 55.0 \\
\hline Passive $M_{\text {MAX }}(\mathrm{mV})$ & $5.85 \pm 2.65$ & $6.51 \pm 2.83$ & 0.843 & $0.599-0.943$ & $1.55(25.02)$ & 14.93 & 4.29 \\
\hline Active $\mathrm{M}_{\mathrm{MAX}}(\mathrm{mV})$ & $6.52 \pm 3.58$ & $6.39 \pm 3.80$ & 0.896 & $0.728-0.962$ & $1.67(25.75)$ & 19.43 & 4.62 \\
\hline $\mathrm{H}_{\mathrm{MAX}}(\mathrm{mV})$ & $1.75 \pm 1.20$ & $1.88 \pm 1.07$ & 0.803 & $0.528-0.926$ & $0.715(39.36)$ & 24.99 & 1.98 \\
\hline H:M Ratio (\%) & $35.27 \pm 22.78$ & $34.65 \pm 23.18$ & 0.860 & $0.643-0.949$ & $0.122(34.79)$ & 23.99 & 0.337 \\
\hline
\end{tabular}

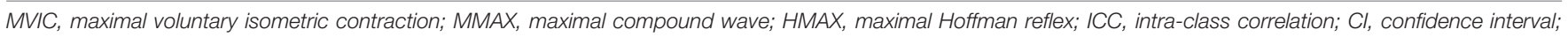
SEm, standard error of measurement; CV, coefficient of variation; MDC, minimal detectable change; $N$, newtons; and mV, millivolts. 
A

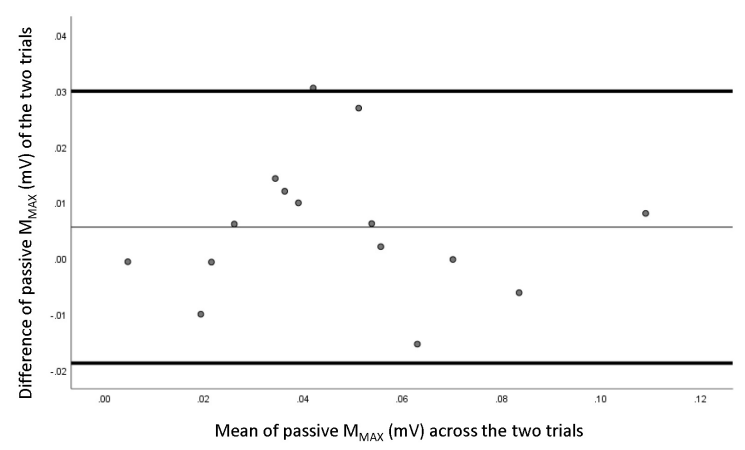

C

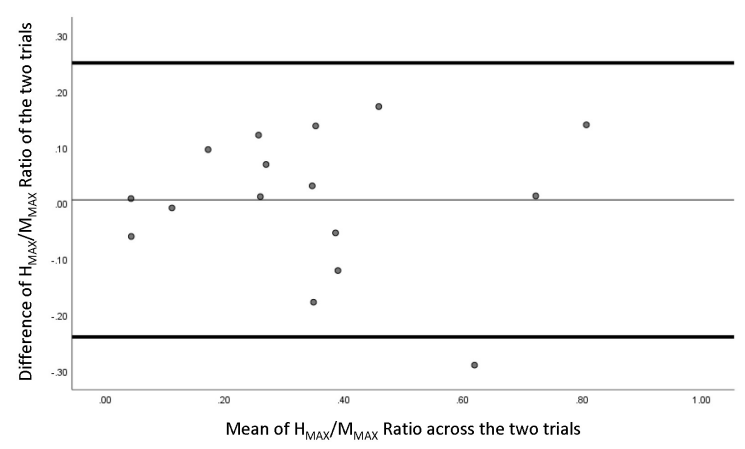

B

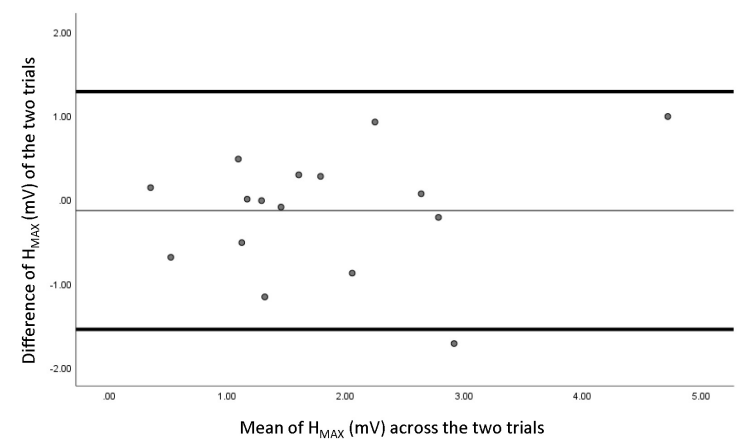

FIGURE 2 | Bland-Altman plot of peripheral measures across the two testing sessions. (A) Resting maximal compound wave, (B) maximal Hoffman reflex, and (C) maximal Hoffman reflex normalized with maximal compound wave.

excellent" relative reliability (ICC $=0.599-0.943)$, a SEm of 1.55 , a CV of $14.93 \%$, and a MDC of 4.29 . Active MMAX had "good to excellent" relative reliability (ICC $=0.728-0.962$ ), a SEm of 1.67 , a CV of $19.43 \%$, and a MDC of 4.62. HMAX had "fair to excellent" relative reliability $(\mathrm{ICC}=0.528-0.926)$, a SEm of 0.715 , a CV of 24.99\%, and a MDC of 1.98. HMAX:MMAX (H:M) ratio had "good to excellent" relative reliability $(\mathrm{ICC}=0.643-0.949)$, a SEm of 0.122 , a CV of $23.99 \%$, and a MDC of 0.337 .

\section{Single-Pulse Transcranial Stimulation}

Reliability data of corticospinal measures are presented in Table 2. Figure 3 depicts the comparison of individual measurements across the two trials. MEPpassive had "good to excellent" relative reliability (ICC $=0.691-0.961$ ), a SEm of 0.0132 , a CV of $17.45 \%$, and MDC of 0.0365 . MEPactive had "fair to excellent" relative reliability (ICC $=0.575-0.937$ ), a SEm of 0.0581 , a CV of $17.45 \%$, and a MDC of 0.161 . CSP had "good to excellent" relative reliability (ICC $=0.663-0.951)$, SEm of 14.4 , a CV of 7.24\%, and a MDC of 40.0. CSP/MEP ratio had "excellent" relative reliability (ICC $=0.793-0.972$ ), a SEm of 35.8 , a CV of $9.68 \%$, and a MDC of 99.1 .

\section{Paired-Pulse Transcranial Stimulation}

Reliability data of intra-cortical measures are presented in Table 2. Figure 4 depicts the comparison of individual measurements across the two trials. SICIpassive had "good to excellent" relative reliability (ICC $=0.666-0.956)$, SEm of 0.0724 , a CV of $22.85 \%$, and a MDC of 0.201 . SICIactive had "fair to excellent" relative reliability (ICC $=0.480-0.919$ ), a SEm of 0.111 , a CV of $17.14 \%$, and a MDC of 0.308 . ICF had "fair to excellent" relative reliability (ICC $=0.420-0.908)$, a SEm of 0.620 , a CV of $19.22 \%$, and a MDC of 1.72 .

\section{DISCUSSION}

The aim of this study was to investigate the reliability of transcranial magnetic and peripheral nerve stimulation outcome measures in the VM. Specifically, we assessed the testretest relative and absolute reliability of peripheral (MVIC, MMAX, and H-reflex), corticospinal (MEP and CSP), and cortical (SICI and ICF) responses. Our findings suggest that the peripheral nerve measures are the most reliable. Corticospinal responses, specifically MEPpassive, CSP, and CSP/MEP, demonstrated "good to excellent" relative reliability whilst active MEP demonstrated the lowest reliability. For intracortical responses, the relative reliability of SICIpassive was "good to excellent." In contrast, ICF and SICIactive demonstrated "poor to excellent" reliability. The findings of this investigation suggest that non-invasive cortical and peripheral nerve stimulation of the VM offer reasonable intra-participant and inter-session reliability in most measures of neurophysiological function. 
TABLE 2 | Reliability data for all corticospinal and intracortical measures across two testing sessions.

\begin{tabular}{|c|c|c|c|c|c|c|c|}
\hline & Day 1 & Day 2 & ICC & $95 \% \mathrm{Cl}$ & SEm (\%) & CV (\%) & MDC \\
\hline $\mathrm{MEP}_{\text {passive }}$ & $0.050 \pm 0.03$ & $0.045 \pm 0.03$ & 0.887 & $0.691-0.961$ & $0.0132(27.82)$ & 17.45 & 0.0365 \\
\hline $\mathrm{MEP}_{\text {active }}$ & $0.14 \pm 0.11$ & $0.14 \pm 0.09$ & 0.830 & $0.575-0.937$ & 0.0581 (41.52) & 19.69 & 0.161 \\
\hline CSP (ms) & $119.26 \pm 32.73$ & $116.73 \pm 31.27$ & 0.866 & $0.663-0.951$ & $14.4(12.24)$ & 7.24 & 40.0 \\
\hline CSP/MEP & $197.53 \pm 116.85$ & $1 S 5.41 \pm 108.65$ & 0.921 & $0.793-0.972$ & $35.8(18.67)$ & 9.68 & 99.1 \\
\hline $\mathrm{SICl}_{\text {passive }}$ & $0.19 \pm 0.14$ & $0.20 \pm 0.15$ & 0.874 & $0666-0.956$ & $0.0724(36.60)$ & 22.85 & 0.201 \\
\hline $\mathrm{SICl}_{\text {active }}$ & $0.40 \pm 0.16$ & $0.41 \pm 0.17$ & 0.783 & 0480-0.919 & $0.111(27.15)$ & 17.14 & 0.308 \\
\hline ICF & $1.60 \pm 1.03$ & $1.39 \pm 0.68$ & 0.751 & $0.420-0.908$ & 0.620 (41.53) & 19.22 & 1.72 \\
\hline
\end{tabular}

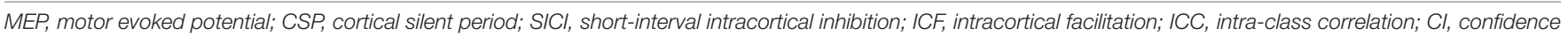
interval; SEm, standard error of measurement; CV, coefficient of variation; and MDC, minimal detectable change.

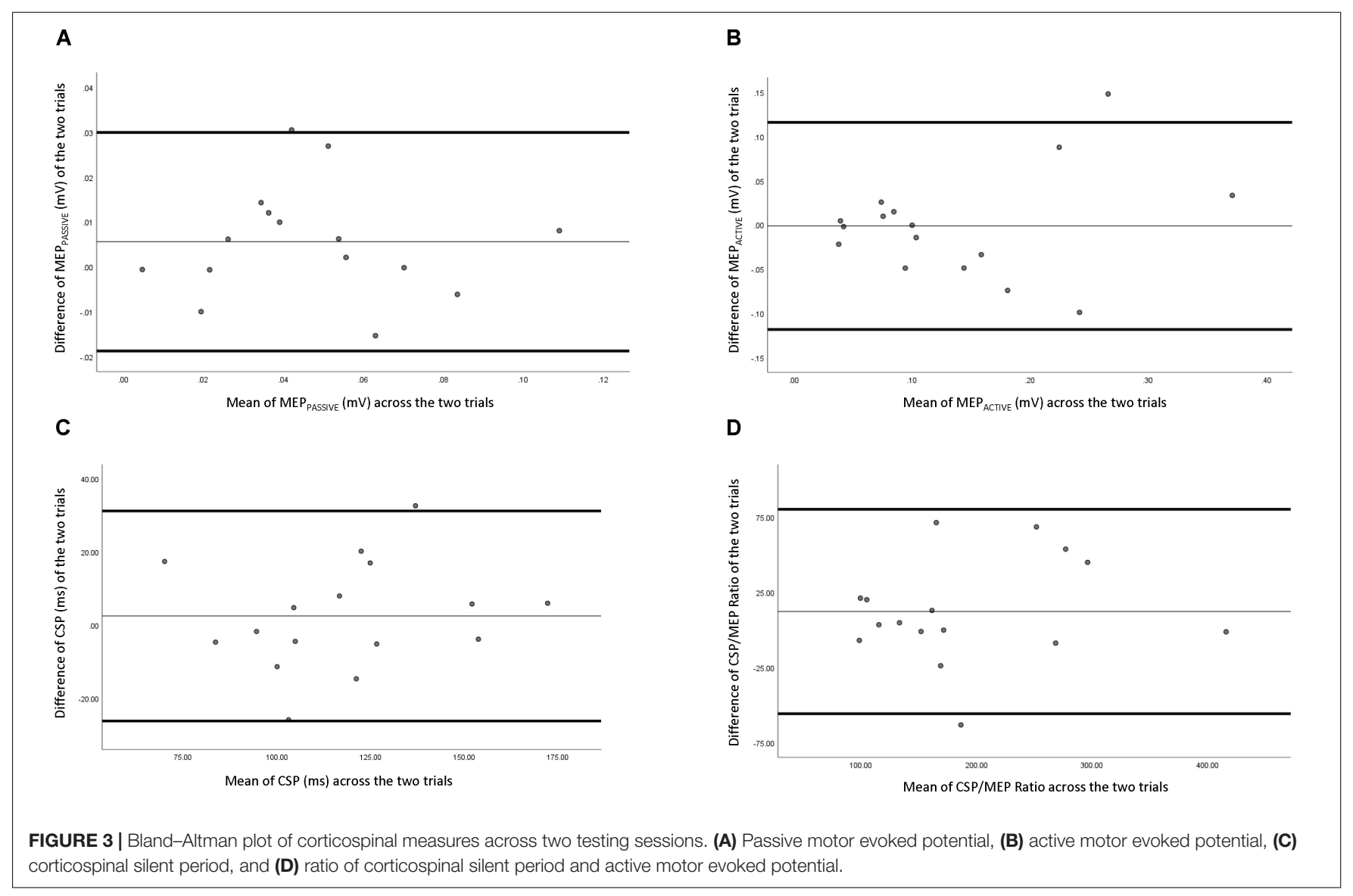

\section{Peripheral Nerve and Spinal Excitability}

The findings demonstrated that the spinal reflex afferent and efferent pathways can be reliably measured in the VM. Our results showed "good to excellent" intra- and inter-participant relative reliability for active MMAX and $\mathrm{H}: \mathrm{M}$ ratio. These findings are similar to those of Hopkins and Wagie (2003), who showed acceptable reliability of the VM H-reflex over six testing sessions. It is worth noting that $\mathrm{H}: \mathrm{M}$ ratio was more reliable than HMAX, indicating the importance to normalize HMAX with MMAX when evaluating spinal excitability. In terms of absolute reliability, $\mathrm{H}$-reflex measurements displayed a higher measurement error (SEm > 30\%) compared to MMAX measurements (SEm $<26 \%$ ). However, the within-subject variation for the resting $\mathrm{H}$-reflex $(\mathrm{CV}=24.99 \%)$ in our study is considerably lower than those reported by Doguet and Jubeau (2014) (CV $\geq 52.2 \%$ ). This large discrepancy between the studies may be due to the different interstimulus intervals used: $20 \mathrm{~s}$ versus the $10 \mathrm{~s}$ interval used by Doguet and Jubeau (2014). The cumulative effects of short repeated stimulation potentially influences monosynaptic reflex activity, due to fatigue or potentiation. Given that fatigue is unlikely to have developed in either condition, the difference may be attributed to a potentiation effect or the longer muscle length ( 75 degrees of knee flexion) used by Doguet and Jubeau (2014). In particular, this lengthened position can induce spinal excitability disturbances due to increased muscle spindle firing (Duclay and Martin, 


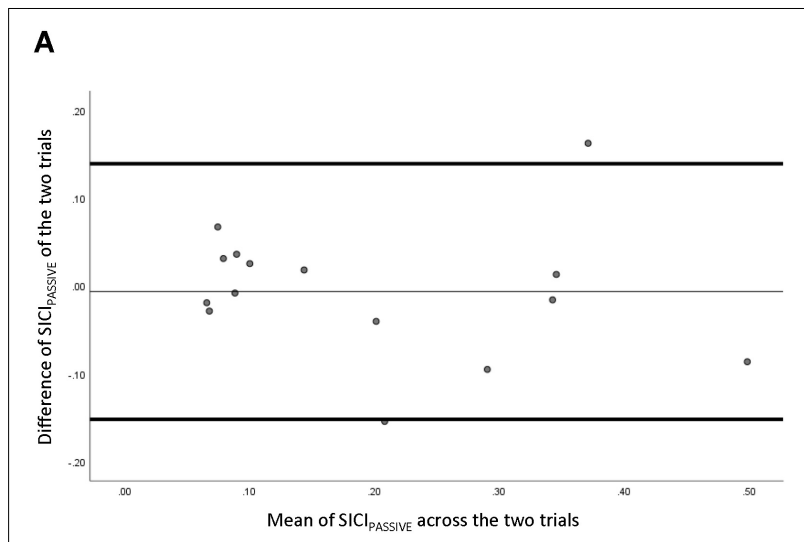

C

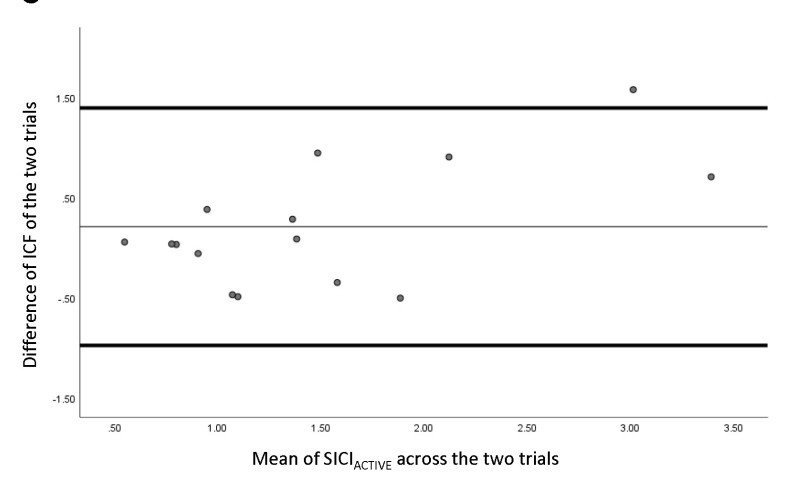

B

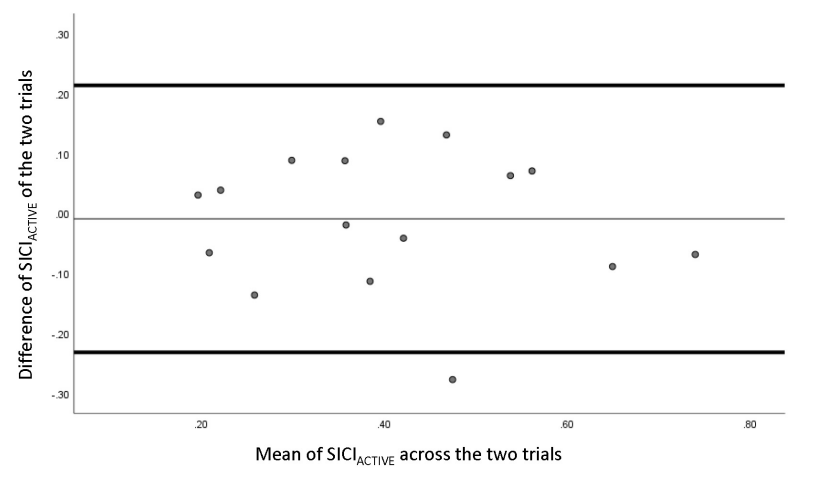

FIGURE 4 | Bland-Altman plot of intracortical measures across two testing sessions. (A) Passive short-interval intracortical inhibition, (B) active short intracortical inhibition, and $(\mathbf{C})$ intracortical facilitation.

2005). Therefore, we conclude that the H-reflex and MMAX can provide a reliable measurement of monosynaptic afferent reflex pathways and motor pool activation when external factors are carefully controlled for. Researchers should therefore consider factors that may influence spinal and lower motor neuron excitability when conducting neurophysiological assessment of the VM. These factors include participants' posture, knee flexion angle, and interstimulus interval used. To allow for feasible session duration and a broad spectrum of neurophysiological assessments (peripheral, corticospinal, and intracortical), we did not collect full input-output curves for any of the measures. See Supplementary Data Sheet 1 for example of modified inputoutput curve and raw H-reflex traces elicited by peripheral nerve stimulation. This must be acknowledged as a limitation, as previous work has highlighted the value of investigating the entire H-reflex and M-wave recruitment curve (Brinkworth et al., 2007; Tucker et al., 2005). We recommend that researchers consider factors such as session duration, participant burden, and expected longevity of the neuromodulatory intervention, alongside the relative and absolute reliability of the measures when determining the appropriate testing protocols.

\section{Corticospinal Excitability and Inhibition}

The current research demonstrated "good to excellent" relative reliability of the MEPpassive, CSP, and CSP/MEP ratio in the VM. The MEPpassive $(\mathrm{ICC}=0.691-0.961)$ proved to be a more reliable measure compared to the "fair to excellent" relative reliability of MEPactive (ICC $=0.575-0.937$ ). This is in line with Ngomo et al. (2012), who reported slightly higher relative reliability in the resting muscle. This may be caused by subtle differences in motor unit recruitment during the active condition, despite dynamometer force output appearing constant. For example, minor alterations in joint angle, limb positioning and factors such as muscle fatigue may mean that motor unit recruitment varies, despite force output appearing steady. In terms of absolute reliability, MEPpassive also displayed a lower measurement error than MEPactive. The MEPactive result was similar to that of Latella et al. (2017) who also recorded responses in the leg extensors. Interestingly, this is contrary to previous work by O'Leary et al. (2015) and Luc et al. (2014), who have concluded "excellent" reliability of the active MEP in the knee extensors. It is worth noting that both these studies used a stimulus intensity that exceeded $120 \%$ of AMT. Ngomo et al. (2012) and Luc et al. (2014) suggest that higher stimulation intensities may improve the reliability of active MEPs, with Luc et al. (2014) finding 130 and 140\% of AMT to be more reliable compared to $105-120 \%$ of AMT. In addition, the CSP and CSP/MEP ratio showed "good to excellent" $(\mathrm{ICC}=0.663-0.951)$ and "excellent" $(\mathrm{ICC}=0.793-0.972)$ reliability, respectively, which is consistent with the findings of O'Leary et al. (2015) in the VL. The CSP and CSP/MEP ratio also demonstrated better absolute reliability (SEm < 20\%) 
compared with the MEP responses. CSP is partly mediated by the inhibition of gamma-aminobutyric acidB receptors in both spinal and cortical circuits (Lefaucheur et al., 2008). Although the reliability of the CSP indicates that corticospinal inhibition can be accurately measured, the CSP/MEP ratio may offer a better insight into the relationship between excitability and inhibition (Orth and Rothwell, 2004). Our results suggest that CSP and CSP/MEP ratio are reliable outcome measures when evaluating corticospinal inhibition. Compared with MEPactive, MEPpassive appears to be a more reliable outcome measure when evaluating corticospinal excitability in the VM.

\section{Intracortical Facilitation and Inhibition}

The results demonstrated "good to excellent" and "fair to excellent" relative reliability of the SICIpassive (ICC $=0.666-0.956)$ and SICIactive (ICC $=0.480-0.919)$, respectively. To our knowledge, no study has investigated the reliability of SICIpassive in the VM, potentially due to the difficulty in obtaining large resting MEP responses in the lower limbs. Our results are in agreement with previous work in the upper limb, which suggested SICI should be assessed under passive conditions in the first dorsal interosseous (Ngomo et al., 2012). This may be partially explained by the fact that muscle activity causes a reduction in intracortical inhibition, and increased activation of facilitatory circuits (Ortu et al., 2008). In terms of absolute reliability, SICIactive displayed a lower level of variability compared with SICIpassive. O'Leary et al. (2015) suggested that SICIactive may be less variable than SICIpassive due to the reduced attentional and somatosensory influences. Using a stronger conditioning stimulus (90\% of AMT) increased reliability of SICIactive (O'Leary et al., 2015). This is also supported by (Temesi et al., 2017), which reported "excellent" relative reliability for SICIactive in the VM with a conditioning stimulus of $90 \%$ of AMT. Using a weaker conditioning stimulus may induce less inhibitory interneurons compared to a stronger conditioning stimulus, leading to a less consistent SICI response (O'Leary et al., 2015). Temesi et al. (2017) also reported "excellent" reliability for SICIpassive, but poor reliability for SICIactive in the hand. Although at this stage it is difficult to pinpoint the mechanisms responsible for the differences between the upper and lower limbs, this finding may be at least in part due to the difference in functional connectivity. For example in locomotion, reciprocal inhibition of the opposite homologous muscle is required, therefore inhibitory circuitry may be more prominent in the motor neurons supplying the lower limbs (Chiou et al., 2013; Hendy et al., 2017). As the functional roles and inhibitory responses between the upper and lower limbs are different, testing parameters may need to be modified for specific muscle groups to obtain the most reliable results. Our results, along with previous reports, suggest that SICI with a conditioning stimulus of $90 \%$ of AMT demonstrate the greatest reliability when evaluating intracortical inhibitory mechanisms in the VM. Finally, our findings demonstrated that the ICF was the least reliable (ICC $=0.420-0.908$ ) out of all measures. Similar to previous studies, ICF was less reliable than both resting and active SICI (Maeda et al., 2002;
Fleming et al., 2012; Du et al., 2014; O’Leary et al., 2015), despite methodological differences. For instance, O’Leary et al. (2015) used different conditioning stimulus intensities for their SICI (70, 80, and 90\%) and ICF $(90,100$, and $110 \%)$ measures when establishing reliability. It is known that both SICI and ICF are affected by $\mathrm{GABA}_{\mathrm{A}}$ receptors (Di Lazzaro et al., 2000), however, ICF is also affected by changes in NMDA receptor sensitivity (Hermsen et al., 2016). Thus, it could be speculated that the lower relative reliability of ICF might be due to mechanisms involving NMDA, however, confirmation from pharmaceutical studies is required. Further research to compare the reliability of different conditioning and test stimulus intensities to quantify SICI and ICF is required to confirm the most reliable protocol when evaluating intracortical excitabitory and inhibitory mechanisms in the VM.

While we report that collectively the corticospinal and intracortical measures demonstrated "good" reliability, it is unclear from this study whether adjusting the conditioning and testing stimulus intensities or ISI may have altered the results. A recent study found that using the average of multiple ISIs led to higher relative and absolute reliability for SICI in the hand (Matamala et al., 2018). Additionally, the value of the ICC in reliability studies must be interpreted with caution. While many have tried to place levels to evaluate the magnitude of ICC, it is not possible to set a universal standard of what constitutes a "good" or "excellent" ICC (Charter and Feldt, 2001). The magnitude of the ICC ultimately depends on between-subjects variability. Assuming the same withinsubject variabilities, the higher the between-subjects variability, the larger the ICC value (Weir, 2005). In this study, all the neurological measures exhibit a large degree of betweensubjects variability, which elevates the ICC. To account for this, we used SEm, MDC, and CV, which are measures of absolute reliability (Weir, 2005). Future test-retest reliability studies in the field should report both absolute and relative reliability measures, and seek to develop rigorous guidelines for absolute reliability. This information will contribute to establish a valid, systematic approach to investigating neurophysiological changes associated with human performance and rehabilitation studies.

\section{CONCLUSION}

Collectively the results suggest that H:M Ratio, MEPpassive, CSP, and CSP/MEP ratio can provide reliable assessment of reflex pathways, excitability and inhibition in the VM. ICF in particular should be used with caution, especially when translating findings into neurological outcomes, such as determining mechanisms underpinning acute or adaptive changes in the leg extensors following interventions. In order to establish the most reliable neurological testing protocols, future studies should compare the reliability of peripheral, corticospinal and intracortical measures for different muscle groups, using various stimulus intensities and ISIs. Protocols need to be established and individualized for the VM, since initial protocols developed in the upper limb may not be optimal for lower limb studies. 


\section{ACKNOWLEDGMENTS}

We thank Patrick James Owen and Jackson Fyfe for their contributions to the study.

\section{AUTHOR CONTRIBUTIONS}

$\mathrm{AH}$ and SL developed the conceptual basis and experimental design for this study. HL conducted data collection. HL and

\section{REFERENCES}

Appell, H. J. (1990). Muscular atrophy following immobilisation. Sports Med. 10, 42-58. doi: 10.2165/00007256-199010010-00005

Atkinson, G., and Nevill, A. M. (1998). Statistical methods for assessing measurement error (reliability) in variables relevant to sports medicine. Sports Med. 26, 217-238. doi: 10.2165/00007256-199826040-00002

Avramidis, K., Strike, P. W., Taylor, P. N., and Swain, I. D. (2003). Effectiveness of electric stimulation of the vastus medialis muscle in the rehabilitation of patients after total knee arthroplasty1. Arch. Phys. Med. Rehabil. 84, 1850-1853. doi: 10.1016/S0003-9993(03)00429-5

Bodofsky, E. B. (1999). Contraction-induced upper extremity H reflexes: normative values. Arch. Phys. Med. Rehabil. 80, 562-565. doi: 10.1016/S0003-9993(99) 90200-9

Braddom, R. I., and Johnson, E. W. (1974). Standardization of H reflex and diagnostic use in Sl radiculopathy. Arch. Phys. Med. Rehabil. 55, 161-166.

Brinkworth, R. S. A., Tuncer, M., Tucker, K. J., Jaberzadeh, S., and Türker, K. S. (2007). Standardization of H-reflex analyses. J. Neurosci. Methods 162, 1-7. doi: 10.1016/j.jneumeth.2006.11.020

Calder, K. M., Hall, L. A., Lester, S. M., Inglis, J. G., and Gabriel, D. A. (2005). Reliability of the biceps brachii M-wave. J. Neuroeng. Rehabil. 2:33.

Cavaleri, R., Schabrun, S. M., and Chipchase, L. S. (2017). The number of stimuli required to reliably assess corticomotor excitability and primary motor cortical representations using transcranial magnetic stimulation (TMS): a systematic review and meta-analysis. Syst. Rev. 6:48. doi: 10.1186/s13643-017-0440-8

Cecen, S., Niazi, I. K., Nedergaard, R. W., Cade, A., Allen, K., Holt, K., et al. (2018). Posture modulates the sensitivity of the H-reflex. Exp. Brain Res. 236, 829-835. doi: 10.1007/s00221-018-5182-x

Charter, R. A., and Feldt, L. S. (2001). Meaning of reliability in terms of correct and incorrect clinical decisions: the art of decision making is still alive. J. Clin. Exp. Neuropsychol. 23, 530-537. doi: 10.1076/jcen.23.4.530.1227

Chen, R. (2000). Studies of human motor physiology with transcranial magnetic stimulation. Muscle Nerve 23, S26-S32. doi: 10.1002/1097-4598(2000)999:9<:: AID-MUS6>3.0.CO;2-I

Chiou, S. Y., Wang, R. Y., Liao, K. K., and Yang, Y. R. (2013). Homologous muscle contraction during unilateral movement does not show a dominant effect on leg representation of the ipsilateral primary motor cortex. PLoS One 8:e72231. doi: 10.1371/journal.pone.0072231

Cowan, S. M., Bennell, K. L., Hodges, P. W., Crossley, K. M., and McConnell, J. (2001). Delayed onset of electromyographic activity of vastus medialis obliquus relative to vastus lateralis in subjects with patellofemoral pain syndrome. Arch. Phys. Med. Rehabil. 82, 183-189. doi: 10.1053/apmr.2001.19022

Di Lazzaro, V., Oliviero, A., Meglio, M., Cioni, B., Tamburrini, G., Tonali, P., et al. (2000). Direct demonstration of the effect of lorazepam on the excitability of the human motor cortex. Clin. Neurophysiol. 111, 794-799. doi: 10.1016/S13882457(99)00314-4

Doguet, V., and Jubeau, M. (2014). Reliability of H-reflex in vastus lateralis and vastus medialis muscles during passive and active isometric conditions. Eur. J. Appl. Physiol. 114, 2509-2519. doi: 10.1007/s00421-014-2969-8

Du, X., Summerfelt, A., Chiappelli, J., Holcomb, H. H., and Hong, L. E. (2014). Individualized brain inhibition and excitation profile in response to pairedpulse TMS. J. Mot. Behav. 46, 39-48. doi: 10.1080/00222895.2013.850401

Duclay, J., and Martin, A. (2005). Evoked H-reflex and V-wave responses during maximal isometric, concentric, and eccentric muscle
CL carried out data analysis, interpretation of results, and manuscript preparation. AH, SL, and CL provided supervision and wrote feedback throughout the research process.

\section{SUPPLEMENTARY MATERIAL}

The Supplementary Material for this article can be found online at: https://www.frontiersin.org/articles/10.3389/fpsyg. 2018.01857/full\#supplementary-material

contraction. J. Neurophysiol. 94, 3555-3562. doi: 10.1152/jn.00348. 2005

Earles, D. R., Dierking, J. T., Robertson, C. T., and Koceja, D. M. (2002). Pre-and post- synaptic control of motoneuron excitability in athletes. Med. Sci. Sports Exerc. 34, 1766-1772. doi: 10.1097/00005768-200211000-00012

Fleming, M. K., Sorinola, I. O., Newham, D. J., Roberts-Lewis, S. F., and Bergmann, J. H. (2012). The effect of coil type and navigation on the reliability of transcranial magnetic stimulation. IEEE Trans. Neural. Syst. Rehabil. Eng. 20, 617-625. doi: 10.1109/TNSRE.2012.2202692

Fratini, A., Cesarelli, M., Bifulco, P., and Romano, M. (2009). Relevance of motion artifact in electromyography recordings during vibration treatment. J. Electromyogr. Kinesiol. 19, 710-718. doi: 10.1016/j.jelekin.2008.04.005

Hendy, A. M., Chye, L., and Teo, W. P. (2017). Cross-activation of the motor cortex during unilateral contractions of the quadriceps. Front. Hum. Neurosci. 11:397. doi: $10.3389 /$ fnhum.2017.00397

Hermens, H. J., Freriks, B., Disselhorst-Klug, C., and Rau, G. (2000). Development of recommendations for SEMG sensors and sensor placement procedures. J. Electromyogr. Kinesiol. 10, 361-374. doi: 10.1016/S1050-6411(00)00027-4

Hermsen, A. M., Haag, A., Duddek, C., Balkenhol, K., Bugiel, H., Bauer, S., et al. (2016). Test-retest reliability of single and paired pulse transcranial magnetic stimulation parameters in healthy subjects. J. Neurol. Sci. 362, 209-216. doi: 10.1016/j.jns.2016.01.039

Hopkins, J. T., Ingersoll, C. D., Cordova, M. L., and Edwards, J. E. (2000). Intrasession and intersession reliability of the soleus H-reflex in supine and standing positions. Electromyogr. Clin. Neurophysiol. 40, 89-94.

Hopkins, J. T., and Palmieri, R. (2004). Effects of ankle joint effusion on lower leg function. Clin. J. Sport Med. 14, 1-7. doi: 10.1097/00042752-200401000-00001

Hopkins, J. T., and Wagie, N. C. (2003). Intrasession and intersession reliability of the quadriceps Hoffmann reflex. Electroencephalogr. Clin. Neurophysiol. 43, 85-89.

Hunter, S. K., McNeil, C. J., Butler, J. E., Gandevia, S. C., and Taylor, J. L. (2016). Short- interval cortical inhibition and intracortical facilitation during submaximal voluntary contractions changes with fatigue. Exp. Brain Res. 234 2541-2551. doi: 10.1007/s00221-016-4658-9

Inghilleri, M., Conte, A., Curra, A., Frasca, V., Lorenzano, C., and Berardelli, A. (2004). Ovarian hormones and cortical excitability, an rTMS study in humans. Clin. Neurophysiol. 115, 1063-1068. doi: 10.1016/j.clinph.2003.12.003

Kamen, G. (2004). Reliability of motor-evoked potentials during resting and active contraction conditions. Med. Sci. Sports Exerc. 36, 1574-1579. doi: 10.1249/01. MSS.0000139804.02576.6A

Kameyama, O., Hayes, K. C., and Wolfe, D. (1989). Methodological considerations contributing to variability of the quadriceps H-reflex. Am. J. Phys. Med. Rehabil. 68, 277-282. doi: 10.1097/00002060-198912000-00004

Koo, T. K., and Li, M. Y. (2016). A guideline of selecting and reporting intraclass correlation coefficients for reliability research. J. Chiropr. Med. 15, 155-163. doi: 10.1016/j.jcm.2016.02.012

Krishnan, C., Allen, E. J., and Williams, G. N. (2011). Effect of knee position on quadriceps muscle force steadiness and activation strategies. Muscle Nerve 43, 563-573. doi: 10.1002/mus.21981

Latella, C., Hendy, A. M., Pearce, A. J., VanderWesthuizen, D., and Teo, W. P. (2016). The time-course of acute changes in corticospinal excitability, intracortical inhibition and facilitation following a single-session heavy strength training of the biceps brachii. Front. Hum. Neurosci. 10:607. doi: 10.3389/ fnhum.2016.00607 
Latella, C., Teo, W. P., Harris, D., Major, B., VanderWesthuizen, D., and Hendy, A. M. (2017). Effects of acute resistance training modality on corticospinal excitability, intra- cortical and neuromuscular responses. Eur. J. Appl. Physiol. 117, 2211-2224. doi: 10.1007/s00421-017-3709-7

Lefaucheur, J. P., Lucas, B., Andraud, F., Hogrel, J. Y., Bellivier, F., Del Cul, A., et al. (2008). Inter-hemispheric asymmetry of motor corticospinal excitability in major depression studied by transcranial magnetic stimulation. J. Psychiatr. Res. 42, 389-398. doi: 10.1016/j.jpsychires.2007.03.001

Luc, B. A., Lepley, A. S., Tevald, M. A., Gribble, P. A., White, D. B., and Pietrosimone, B. G. (2014). Reliability of corticomotor excitability in leg and thigh musculature at 14 and 28 days. J. Sport Rehabil. 23, 330-338. doi: 10.1123/ jsr.2013-0069

Maeda, F., Gangitano, M., Thall, M., and Pascual-Leone, A. (2002). Inter-and intra- individual variability of paired-pulse curves with transcranial magnetic stimulation (TMS). Clin. Neurophysiol. 113, 376-382. doi: 10.1016/S13882457(02)00008-1

Matamala, J. M., Howells, J., Dharmadasa, T., Trinh, T., Ma, Y., Lera, L., et al. (2018). Inter-session reliability of short-interval intracortical inhibition measured by threshold tracking TMS. Neurosci. Lett. 674, 18-23. doi: 10.1016/ j.neulet.2018.02.065

Miller, T. A., Newall, A. R., and Jackson, D. A. (1995). H-reflexes in the upper extremity and the effects of voluntary contraction. Electromyogr. Clin. Neurophysiol. 35, 121-128.

Mouthon, A. A., Ruffieux, J., Keller, M., and Taube, W. (2016). Age-related differences in corticospinal excitability during observation and motor imagery of balance tasks. Front. Aging Neurosci. 8:317. doi: 10.3389/fnagi.2016. 00317

Ngomo, S., Leonard, G., Moffet, H., and Mercier, C. (2012). Comparison of transcranial magnetic stimulation measures obtained at rest and under active conditions and their reliability. J. Neurosci. Methods 205, 65-71. doi: 10.1016/j. jneumeth.2011.12.012

Nuzzo, J. L., Trajano, G. S., Barry, B. K., Gandevia, S. C., and Taylor, J. L. (2016). Arm posture-dependent changes in corticospinal excitability are largely spinal in origin. J. Neurophysiol. 115, 2076-2082. doi: 10.1152/jn.00885.2015

O'Leary, T. J., Morris, M. G., Collett, J., and Howells, K. (2015). Reliability of single and paired-pulse transcranial magnetic stimulation in the vastus lateralis muscle. Muscle Nerve 52, 605-615. doi: 10.1002/mus.24584

Orth, M., and Rothwell, J. C. (2004). The cortical silent period: intrinsic variability and relation to the waveform of the transcranial magnetic stimulation pulse. Clin. Neurophysiol. 115, 1076-1082. doi: 10.1016/j.clinph.2003.12.025

Ortu, E., Deriu, F., Suppa, A., Tolu, E., and Rothwell, J. C. (2008). Effects of volitional contraction on intracortical inhibition and facilitation in the human motor cortex. J. Physiol. 586, 5147-5159. doi: 10.1113/jphysiol.2008. 158956

Palmieri, R. M., Hoffman, M. A., and Ingersoll, C. D. (2002). Intersession reliability for $\mathrm{H}$ - reflex measurements arising from the soleus, peroneal, and tibialis anterior musculature. Int. J. Neurosci. 112, 841-850. doi: 10.1080/ 00207450290025851

Palmieri, R. M., Ingersoll, C. D., and Hoffman, M. A. (2004). The Hoffmann reflex: methodologic considerations and applications for use in sports medicine and athletic training research. J. Athl. Train. 39, 268-277.

Pearcey, G. E., Power, K. E., and Button, D. C. (2014). Differences in supraspinal and spinal excitability during various force outputs of the biceps brachii in chronic-and non- resistance trained individuals. PLoS One 9:e98468. doi: 10. 1371/journal.pone.0098468

Peeler, J., and Anderson, J. E. (2007). Structural parameters of the vastus medialis muscle and its relationship to patellofemoral joint deterioration. Clin. Anat. 20, 307-314. doi: 10.1002/ca.20375
Pierrot-Deseilligny, E., and Mazevet, D. (2000). The monosynaptic reflex: a tool to investigate motor control in humans. Interest and limits. Neurophysiol. Clin. 30, 67-80. doi: 10.1016/S0987-7053(00)00062-9

Remaud, A., Bilodeau, M., and Tremblay, F. (2014). Age and muscle-dependent variations in corticospinal excitability during standing tasks. PLoS One 9:110004. doi: 10.1371/journal.pone.0110004

Rossi, S. (2009). A. Pascual-leone and the safety of TMS consensus group, Safety, ethical considerations, and application guidelines for the use of transcranial magnetic stimulation in clinical practice and research. Clin. Neurophysiol. 120, 2008-2039. doi: 10.1016/j.clinph.2009.08.016

Rossini, P. M., Burke, D., Chen, R., Cohen, L. G., Daskalakis, Z., Di Iorio, R., et al. (2015). Non-invasive electrical and magnetic stimulation of the brain, spinal cord, roots and peripheral nerves: basic principles and procedures for routine clinical and research application. An updated report from an IFCN Committee. Clin. Neurophysiol. 126, 1071-1107. doi: 10.1016/j.clinph.2015.02.001

Rothwell, J. C., Hallett, M., Berardelli, A., Eisen, A., Rossini, P., and Paulus, W. (1999). Magnetic stimulation: motor evoked potentials. Electroencephalogr. Clin. Neurophysiol. Suppl. 52, 97-103.

Sacco, P., Thickbroom, G. W., Thompson, M. L., and Mastaglia, F. L. (1997). Changes in corticomotor excitation and inhibition during prolonged submaximal muscle contractions. Muscle Nerve 20, 1158-1166. doi: 10.1002/ (SICI)1097-4598(199709)20:9<1158::AID-MUS11>3.0.CO;2-P

Sale, M. V., Ridding, M. C., and Nordstrom, M. A. (2007). Factors influencing the magnitude and reproducibility of corticomotor excitability changes induced by paired associative stimulation. Exp. Brain Res. 181, 615-626. doi: 10.1007/ s00221-007-0960-x

Taylor, J. L., Todd, G., and Gandevia, S. C. (2006). Evidence for a supraspinal contribution to human muscle fatigue. Clin. Exp. Pharmacol. Physiol. 33, 400-405. doi: 10.1111/j.1440-1681.2006.04363.x

Temesi, J., Ly, S. N., and Millet, G. Y. (2017). Reliability of single-and paired-pulse transcranial magnetic stimulation for the assessment of knee extensor muscle function. J. Neurol. Sci. 375, 442-449. doi: 10.1016/j.jns.2017.02.037

Thomas, A. C., Pietrosimone, B. G., and Bayer, C. J. (2016). Agreement between investigators using paired-pulse transcranial magnetic stimulation to assess quadriceps intracortical excitability. J. Sport Rehabil. 25: jsr.2015-0121. doi: 10.1123/jsr.2015-0121

Tucker, K. J., Tuncer, M., and Türker, K. S. (2005). A review of the H-reflex and M-wave in the human triceps surae. Hum. Mov. Sci. 24, 667-688. doi: 10.1016/j.humov.2005.09.010

Wassermann, E. (2008). Oxford Handbook of Transcranial Stimulation, eds C. Epstein, U. Ziemann, and E. Wassermann (Oxford: Oxford University Press).

Wassermann, E. M. (2002). Variation in the response to transcranial magnetic brain stimulation in the general population. Clin. Neurophysiol. 113, 1165-1171. doi: 10.1016/S1388-2457(02)00144-X

Weir, J. P. (2005). Quantifying test-retest reliability using the intraclass correlation coefficient and the SEM. J. Strength Cond. Res. 19, 231-240.

Conflict of Interest Statement: The authors declare that the research was conducted in the absence of any commercial or financial relationships that could be construed as a potential conflict of interest.

Copyright (c) 2018 Leung, Latella, Lamon and Hendy. This is an open-access article distributed under the terms of the Creative Commons Attribution License (CC BY). The use, distribution or reproduction in other forums is permitted, provided the original author(s) and the copyright owner(s) are credited and that the original publication in this journal is cited, in accordance with accepted academic practice. No use, distribution or reproduction is permitted which does not comply with these terms. 